\title{
TITLE:
}

\section{Some properties of energy measures on Sierpinski gasket type fractals}

\author{
AUTHOR(S):
}

Hino, Masanori

\section{CITATION:}

Hino, Masanori. Some properties of energy measures on Sierpinski gasket type fractals. Journal of Fractal Geometry 2016, 3(3): 245-263

\section{ISSUE DATE:}

2016-09-14

URL:

http://hdl.handle.net/2433/231411

\section{RIGHT:}

This is the accepted version of the article, which has been published in final form at http://doi.org/10.4171/JFG/36. The full-text file will be made open to the public on 14 September 2016 in accordance with publisher's 'Terms and

Conditions for Self-Archiving'. This is not the published version. Please cite only the published version. この論文は出版 社版でありません。引用の際には出版社版をご確認ご利用ください。 


\title{
Some properties of energy measures on Sierpinski gasket type fractals
}

\author{
Masanori Hino* \\ Dedicated to Professor Shinzo Watanabe on the occasion of his 80th birthday
}

\begin{abstract}
We confirm, in a more general framework, a part of the conjecture posed by R. Bell, C.-W. Ho, and R. S. Strichartz [Energy measures of harmonic functions on the Sierpiński gasket, Indiana Univ. Math. J. 63 (2014), 831-868] on the distribution of energy measures for the canonical Dirichlet form on the two-dimensional standard Sierpinski gasket.
\end{abstract}

Mathematics Subject Classification (2010). 28A80; 31C25, 60B20.

Keywords. energy measure, Dirichlet form, Sierpinski gasket, self-similar set.

\section{Introduction}

Energy measures associated with strong local regular Dirichlet forms describe certain local structures of Dirichlet forms. For the standard energy form on a Euclidean space, the energy measure of a function $f$ is given explicitly by $|\nabla f(x)|^{2} d x$. On the other hand, for canonical Dirichlet forms on fractals, energy measures do not usually have simple expressions and it seems a difficult problem to know how they are distributed in the state space. For example, energy measures are singular with respect to self-similar measures for self-similar Dirichlet forms on most self-similar fractals [2, 7, 12, 14]. Recently, Bell, Ho, and Strichartz [1] studied several properties of energy measures associated with the canonical Dirichlet form on the two-dimensional standard Sierpinski gasket. In particular, they introduced non-negative coefficients $\left\{b_{j}^{(w)}\right\}_{j=1}^{3}$ for describing the distribution of the energy measures of harmonic functions on the cells corresponding to each word $w$ (see Section 2 for details), and posed conjectures about properties of the limiting behavior of $\left\{b_{j}^{(w)}\right\}_{j=1}^{3}$ as $w$ converges to an infinite sequence of words ([1, Conjectures 7.1 and 7.2$]$ ). In this paper, we confirm a part of the conjecture in a slightly

*This research was partially supported by JSPS KAKENHI Grant Number 24540170. 
M. Hino

generalized setting. The proof suggests that the conjectured properties depend strongly on the fractals under consideration having three vertices. Our approach is more straightforward than the original one [1]: we use only primitive linear operators for the analysis and utilize some results on limits of random matrices (cf. $[3,8-10,14]$ ).

The remainder of this paper is organized as follows. In Section 2, we provide a framework for Dirichlet forms on self-similar sets and give some preliminary results. The conjectures in [1] are also stated. In Section 3, we prove the main theorem. Section 4 provides some concluding remarks and discussions.

\section{Framework and preliminaries}

We first introduce a class of self-similar sets and the Dirichlet forms defined on them, following [13]. Let $K$ be a compact, connected, and metrizable space. Let $\left\{\psi_{i}\right\}_{i \in S}$ be a family of continuous injective mappings from $K$ to itself having a finite index set $S$ with $\# S \geq 2$. Denote $S^{\mathbb{N}}$ by $\Sigma$ and each element of $\Sigma$ by $\omega_{1} \omega_{2} \omega_{3} \cdots$ with $\omega_{n} \in S$ for every $n \in \mathbb{N}$. For $i \in S$, a shift operator $\sigma_{i}: \Sigma \rightarrow \Sigma$ is defined by $\sigma_{i}\left(\omega_{1} \omega_{2} \cdots\right)=i \omega_{1} \omega_{2} \cdots$. We assume that there exists a continuous surjective map $\pi: \Sigma \rightarrow K$ such that $\psi_{i} \circ \pi=\pi \circ \sigma_{i}$ for each $i \in S$. The triplet $\left(K, S,\left\{\psi_{i}\right\}_{i \in S}\right)$ is then called a self-similar structure. Define $W_{m}=S^{m}$ for $m \in \mathbb{Z}_{+}:=\mathbb{N} \cup\{0\}$ and denote $\bigcup_{m \in \mathbb{Z}_{+}} W_{m}$ by $W_{*}$. For $w=w_{1} w_{2} \cdots w_{m} \in W_{m} \subset W_{*}$, we define $\psi_{w}=\psi_{w_{1}} \circ \psi_{w_{2}} \circ \cdots \circ \psi_{w_{m}}$ and $K_{w}=\psi_{w}(K)$. For $w=w_{1} \cdots w_{m} \in W_{m}$ and $w^{\prime}=w_{1}^{\prime} \cdots w_{n}^{\prime} \in W_{n}, w w^{\prime}$ denotes $w_{1} \cdots w_{m} w_{1}^{\prime} \cdots w_{n}^{\prime} \in W_{m+n}$. We set

$$
\mathcal{P}=\bigcup_{m=1}^{\infty} \sigma^{m}\left(\pi^{-1}\left(\bigcup_{i, j \in S, i \neq j}\left(K_{i} \cap K_{j}\right)\right)\right) \quad \text { and } \quad V_{0}=\pi(\mathcal{P}),
$$

where $\sigma^{m}: \Sigma \rightarrow \Sigma$ is defined by $\sigma^{m}\left(\omega_{1} \omega_{2} \cdots\right)=\omega_{m+1} \omega_{m+2} \cdots$. We assume that $\mathcal{P}$ is a finite set. In such a case, $\left(K, S,\left\{\psi_{i}\right\}_{i \in S}\right)$ is called post-critically finite. Then, from [13, Lemma 1.3.14], each $\psi_{i}$ has a unique fixed point $\pi(i i i \cdots)$.

For a finite set $V$, let $l(V)$ denote the space of all real-valued functions on $V$. A canonical inner product $(\cdot, \cdot)_{l(V)}$ on $l(V)$ is defined by $(u, v)_{l(V)}=\sum_{p \in V} u(p) v(p)$. The associated norm is denoted by $|\cdot|_{l(V)}$. Let $D=\left(D_{p q}\right)_{p, q \in V_{0}}$ be a symmetric linear operator on $l\left(V_{0}\right)$ with the following properties.

(D1) $D$ is non-positive definite;

(D2) $D u=0$ if and only if $u$ is constant on $V_{0}$;

(D3) $D_{p q} \geq 0$ for all $p, q \in V_{0}$ with $p \neq q$.

Define $\mathcal{E}^{(0)}(u, v)=(-D u, v)_{l\left(V_{0}\right)}$ for $u, v \in l\left(V_{0}\right)$. For $m \in \mathbb{N}$, let $V_{m}=\bigcup_{w \in W_{m}} \psi_{w}\left(V_{0}\right)$. For $\boldsymbol{r}=\left\{r_{i}\right\}_{i \in S}$ with $r_{i}>0$ for all $i \in S$, define a bilinear form $\mathcal{E}^{(m)}$ on $l\left(V_{m}\right)$ by

$$
\mathcal{E}^{(m)}(u, v)=\sum_{w \in W_{m}} \frac{1}{r_{w}} \mathcal{E}^{(0)}\left(\left.u \circ \psi_{w}\right|_{V_{0}},\left.v \circ \psi_{w}\right|_{V_{0}}\right), \quad u, v \in l\left(V_{m}\right),
$$



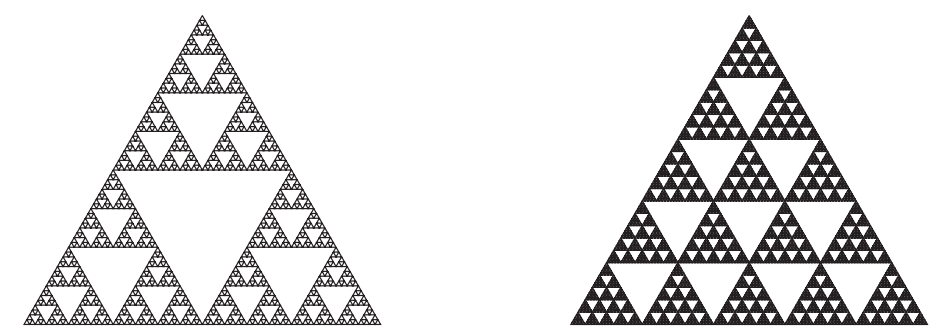

Figure 1. Two-dimensional level $l$ Sierpinski gaskets $S G_{l}(l=2,5)$

where $r_{w}=\prod_{i=1}^{m} r_{w_{i}}$ for $w=w_{1} w_{2} \cdots w_{m} \in W_{m}$. The pair $(D, \boldsymbol{r})$ is called a harmonic structure if

$$
\mathcal{E}^{(0)}(u, u)=\inf \left\{\mathcal{E}^{(1)}(v, v)\left|v \in l\left(V_{1}\right), v\right|_{V_{0}}=u\right\} \quad \text { for every } u \in l\left(V_{0}\right) .
$$

Then, for $m \geq 0$, the identity

$$
\mathcal{E}^{(m)}(u, u)=\inf \left\{\mathcal{E}^{(m+1)}(v, v)\left|v \in l\left(V_{m+1}\right), v\right|_{V_{m}}=u\right\}
$$

holds for every $u \in l\left(V_{m}\right)$. If, moreover, $0<r_{i}<1$ for all $i \in S$, the harmonic structure is called regular. Henceforth, we assume that a regular harmonic structure $(D, \boldsymbol{r})$ is given. Let $\mu$ be a finite Borel measure on $K$ with full support. Then

$$
\mathcal{E}(u, v)=\lim _{m \rightarrow \infty} \mathcal{E}^{(m)}\left(\left.u\right|_{V_{m}},\left.v\right|_{V_{m}}\right), \quad u, v \in \mathcal{F}
$$

with

$$
\mathcal{F}=\left\{u \in C(K) \subset L^{2}(K, \mu) \mid \sup _{m \in \mathbb{Z}_{+}} \mathcal{E}^{(m)}\left(\left.u\right|_{V_{m}},\left.u\right|_{V_{m}}\right)<\infty\right\}
$$

defines a bilinear form $(\mathcal{E}, \mathcal{F})$ on $L^{2}(K, \mu)$ associated with $(D, \boldsymbol{r})$. Under a mild condition on $\mu,(\mathcal{E}, \mathcal{F})$ becomes a strong local regular Dirichlet form on $L^{2}(K, \mu)$ (self-similar measures are adequate, for example; see [13, Theorem 3.4.6]). We will assume such a $\mu$ is chosen; the choice is not important for subsequent arguments. We always take continuous functions as $\mu$-versions of elements of $\mathcal{F}$.

Example 2.1. Typical examples are two-dimensional level $l$ Sierpinski gaskets $S G_{l}$ for $l \geq 2$, which are realized by compact subsets of $\mathbb{R}^{2}$ (see Figure 1 ). They are constructed by $l(l+1) / 2$ contraction mappings $\psi_{i}$ defined as $\psi_{i}(z)=l^{-1} z+b_{l, i}$ with suitable $b_{l, i} \in \mathbb{R}^{2}$ and characterized by nonempty compact subsets satisfying $S G_{l}=\bigcup_{i=1}^{l(l+1) / 2} \psi_{i}\left(S G_{l}\right)$. We call $S G_{2}$ the two-dimensional standard Sierpinski gasket. The set $V_{0}$ consists of the three vertices $p_{1}, p_{2}$, and $p_{3}$ of the largest triangle in $S G_{l}$. We renumber $\left\{\psi_{i}\right\}_{i=1}^{l(l+1) / 2}$ so that $\psi_{i}$ denotes the contraction mapping whose fixed point is $p_{i}$ for $i=1,2,3$ and define the matrix $D$ by

$$
D=\left(D_{p_{i} p_{j}}\right)_{i, j=1}^{3}=\left[\begin{array}{ccc}
-2 & 1 & 1 \\
1 & -2 & 1 \\
1 & 1 & -2
\end{array}\right] \text {. }
$$


Then, there exists a unique number $r$ such that $0<r<1$ and $(D, \boldsymbol{r})$ is a regular harmonic structure with $\boldsymbol{r}=\{r, \ldots, r\}$. This example satisfies the conditions (A1), (A3), and (A4) that are stated later. If we take the normalized Hausdorff measure as $\mu$, the diffusion process associated with the Dirichlet form as stated above is regarded as the Brownian motion on $S G_{l}$.

We now resume our discussion of the general situation. For each $x \in l\left(V_{0}\right)$, there exists a unique function $h \in \mathcal{F}$ such that $\left.h\right|_{V_{0}}=x$ and $h$ attains the infimum of $\left\{\mathcal{E}(g, g) \mid g \in \mathcal{F}\right.$ and $\left.\left.g\right|_{V_{0}}=x\right\}$. Such a function $h$ is called a harmonic function and the totality of $h$ is denoted by $\mathcal{H}$. The map $\iota: l\left(V_{0}\right) \ni x \mapsto h \in \mathcal{H}$ is linear, so we can identify $\mathcal{H}$ with $l\left(V_{0}\right)$ by this map. For $i \in S$, we define a linear operator $A_{i}: l\left(V_{0}\right) \rightarrow l\left(V_{0}\right)$ by $\left(A_{i} x\right)(p)=(\iota(x))\left(\psi_{i}(p)\right)$ for $x \in l\left(V_{0}\right)$ and $p \in V_{0}$. For $w=w_{1} w_{2} \cdots w_{m} \in W_{m}$, we set $A_{w}=A_{w_{m}} A_{w_{m-1}} \cdots A_{w_{1}}$. With an abuse of notation, $D$ and $A_{w}$ can also be considered as linear maps from $\mathcal{H}$ to $l\left(V_{0}\right)$ by identifying $\mathcal{H}$ with $l\left(V_{0}\right)$. Let $\mathbf{1} \in l\left(V_{0}\right)$ denote a constant function on $V_{0}$ with value 1 . Set

$$
\tilde{l}\left(V_{0}\right)=\left\{x \in l\left(V_{0}\right) \mid(x, \mathbf{1})_{l\left(V_{0}\right)}=0\right\}
$$

and let $P: l\left(V_{0}\right) \rightarrow l\left(V_{0}\right)$ be the orthogonal projection onto $\tilde{l}\left(V_{0}\right)$.

Lemma 2.2. The range of $D$ is $\tilde{l}\left(V_{0}\right)$.

Proof. For $x \in l\left(V_{0}\right)$,

$$
(D x, \mathbf{1})_{l\left(V_{0}\right)}=(x, D \mathbf{1})_{l\left(V_{0}\right)}=0 .
$$

Therefore, $D\left(l\left(V_{0}\right)\right) \subset \tilde{l}\left(V_{0}\right)$. Since the dimensions of $D\left(l\left(V_{0}\right)\right)$ and $\tilde{l}\left(V_{0}\right)$ are both $\# V_{0}-1$, we obtain the result.

For $f \in \mathcal{F}, \nu_{f}$ denotes the energy measure of $f$ (cf. [6]); in our situation, $\nu_{f}$ is the unique finite Borel measure on $K$ such that

$$
\int_{K} g d \nu_{f}=2 \mathcal{E}(f, f g)-\mathcal{E}\left(f^{2}, g\right) \quad \text { for all } g \in \mathcal{F} .
$$

In particular, the energy measures of the constant functions are the zero measure in our framework. From the general theory, it is known that every energy measure for strong local regular Dirichlet forms does not have a point mass (from the energy image density property; see, e.g., [5, Theorem 4.3.8] or [4, Theorem I.7.1.1] for the proof). Therefore, energy measures $\nu_{f}$ do not have a mass on the countable set $V_{*}:=\bigcup_{m \in \mathbb{Z}_{+}} V_{m}$ for any $f \in \mathcal{F}$. A concrete expression for $\nu_{f}$ with $f \in \mathcal{H}$ can be provided as follows.

Lemma 2.3 (cf. [12, Lemma 4]). For any $f \in \mathcal{H}$ and $w \in W_{*}$,

$$
\nu_{f}\left(K_{w}\right)=-{\frac{2}{r_{w}}}^{t}\left(A_{w} f\right) D\left(A_{w} f\right)
$$


We remark that the right-hand side of $(2.1)$ is also described as $\left(2 / r_{w}\right) \mathcal{E}(f \circ$ $\left.\psi_{w}, f \circ \psi_{w}\right)$. This expression justifies an intuitive meaning of $\nu_{f}\left(K_{w}\right)$ as a "local energy of $f$ on $K_{w}$." *

The mutual energy measure $\nu_{f, g}$ for $f$ and $g$ in $\mathcal{F}$ is a signed measure on $K$ defined by $\nu_{f, g}=\left(\nu_{f+g}-\nu_{f}-\nu_{g}\right) / 2$. For every Borel subset $B$ of $K$, the inequality

$$
\left|\nu_{f, g}(B)\right|^{2} \leq \nu_{f}(B) \nu_{g}(B)
$$

holds.

We assume the following condition.

(A1) Each point of $V_{0}$ is a fixed point of some $\psi_{j}$. More precisely, there exists a subset $S_{0}$ of $S$ such that $\# S_{0}=\# V_{0}$ and, for each $p \in V_{0}$, there exists $j \in S_{0}$ such that $\psi_{j}(p)=p$. Furthermore, $K \backslash\{p\}$ is a connected set for every $p \in V_{0}$.

Under this condition, we have the following two lemmas.

Lemma 2.4 (cf. [13, Theorem A.1.2] and [12, Lemma 5]). Let $j \in S_{0}$. Take $p \in V_{0}$ such that $\psi_{j}(p)=p$ and let $u_{j}$ denote the column vector $\left(D_{q p}\right)_{q \in V_{0}}$. Then

(1) $r_{j}$ is a simple eigenvalue of $A_{j}$ and ${ }^{t} A_{j}$. Moreover, the modulus of all the eigenvalues of $A_{j}$ and ${ }^{t} A_{j}$ other than 1 and $r_{j}$ are less than $r_{j}$.

(2) The vector $u_{j}$ belongs to $\tilde{l}\left(V_{0}\right)$ and is an eigenvector of ${ }^{t} A_{j}$ with respect to the eigenvalue $r_{j}$.

(3) There is a unique eigenvector $v_{j}$ of $A_{j}$ with respect to the eigenvalue $r_{j}$ such that $\left(u_{j}, v_{j}\right)_{l\left(V_{0}\right)}=1$. Moreover, every component of $v_{j}$ is non-negative.

Lemma 2.5 (cf. [12, Lemma 6]). For $j \in S_{0}$ and $x \in l\left(V_{0}\right)$,

$$
\lim _{n \rightarrow \infty} r_{j}^{-n} P A_{j}^{n} x=\left(u_{j}, x\right)_{l\left(V_{0}\right)} P v_{j} .
$$

Let $h_{1}, \ldots, h_{N}(N \in \mathbb{N})$ be a finite number of harmonic functions such that $\mathcal{H}$ is spanned by $h_{1}, \ldots, h_{N}$ and constant functions. We denote $\sum_{k=1}^{N} \nu_{h_{k}}$ by $\nu$.

Lemma 2.6. For every $f \in \mathcal{H}, \nu_{f}$ is absolutely continuous with respect to $\nu$.

Proof. For some $\alpha_{i} \in \mathbb{R}(i=1, \ldots, N)$ and $\beta \in \mathbb{R}, f$ can be written as $f=$ $\sum_{i=1}^{N} \alpha_{i} h_{i}+\beta$. Then, $\nu_{f}=\sum_{i, j=1}^{N} \alpha_{i} \alpha_{j} \nu_{h_{i}, h_{j}}$. By (2.2), this is absolutely continuous with respect to $\nu$.

We further assume the following condition.

(A2) For every $i \in S, A_{i}$ is invertible.

${ }^{*}$ Compare this expression also with the following classical situation: for a Dirichlet form $\left(Q, H^{1}\left(\mathbb{R}^{d}\right)\right)$ on $L^{2}\left(\mathbb{R}^{d}, d x\right)$, where $Q(f, g)=(1 / 2) \int_{\mathbb{R}^{d}}(\nabla f, \nabla g)_{\mathbb{R}^{d}} d x$ for $f, g \in H^{1}\left(\mathbb{R}^{d}\right)$, the energy measure of $f \in H^{1}\left(\mathbb{R}^{d}\right)$ is given by $|\nabla f|_{\mathbb{R}^{d}}^{2} d x$. 
For $S G_{l}$ with the canonical harmonic structure in Example 2.1, (A2) has been confirmed for $l \leq 50$ by numerical computation (cf. [9, p. 297]). It is conjectured that it is true for all $l \geq 2$.

Lemma 2.7. For $j \in S_{0}$ and $w \in W_{*},{ }^{t} A_{w} u_{j}$ belongs to $\tilde{l}\left(V_{0}\right)$ and is nonzero.

Proof. This is clear because of the identity $A_{w} \mathbf{1}=\mathbf{1}$ and Condition (A2).

For $j \in S_{0}$ and $w \in W_{*}$, let

$$
a_{j}^{(w)}=\sum_{k=1}^{N}\left(u_{j}, A_{w} h_{k}\right)_{l\left(V_{0}\right)}^{2} .
$$

From Lemma 2.7, $a_{j}^{(w)}$ is strictly positive due to the choice of $\left\{h_{k}\right\}_{k=1}^{N}$.

We have some explicit information on the Radon-Nikodym derivative $d \nu_{f} / d \nu$ for harmonic functions $f$.

Lemma 2.8. For any $f \in \mathcal{H}, w \in W_{*}$, and $j \in S_{0}$,

$$
\lim _{n \rightarrow \infty} \frac{\nu_{f}\left(K_{w j^{n}}\right)}{\nu\left(K_{w j^{n}}\right)}=\frac{\left(u_{j}, A_{w} f\right)_{l\left(V_{0}\right)}^{2}}{a_{j}^{(w)}}
$$

where $K_{w j^{n}}$ denotes $K_{w} \underbrace{j \ldots j}_{n}$.

We denote this limit by $\frac{d \nu_{f}}{d \nu}\left(w j^{\infty}\right)$. Although this precise notation may look like $\frac{d \nu_{f}}{d \nu}\left(\pi\left(w j^{\infty}\right)\right)$, it is not clear whether the relation $\pi\left(w j^{\infty}\right)=\pi\left(\tilde{w} \tilde{j}^{\infty}\right)$ implies the identity $\frac{d \nu_{f}}{d \nu}\left(w j^{\infty}\right)=\frac{d \nu_{f}}{d \nu}\left(\tilde{w} \tilde{j}^{\infty}\right)$.

Proof of Lemma 2.8. From Lemma 2.3, Lemma 2.5 and the identity $D={ }^{t} P D P$,

$$
\begin{aligned}
\frac{\nu_{f}\left(K_{w j^{n}}\right)}{\nu\left(K_{w j^{n}}\right)} & =\frac{{ }^{t}\left(P A_{j}^{n} A_{w} f\right) D\left(P A_{j}^{n} A_{w} f\right)}{\sum_{k=1}^{N}{ }^{t}\left(P A_{j}^{n} A_{w} h_{k}\right) D\left(P A_{j}^{n} A_{w} h_{k}\right)} \\
& \stackrel{n \rightarrow \infty}{\longrightarrow} \frac{\left(u_{j}, A_{w} f\right)_{l\left(V_{0}\right)}^{2}{ }^{t}\left(P v_{j}\right) D\left(P v_{j}\right)}{\sum_{k=1}^{N}\left(u_{j}, A_{w} h_{k}\right)_{l\left(V_{0}\right)}^{2}{ }^{t}\left(P v_{j}\right) D\left(P v_{j}\right)}=\frac{\left(u_{j}, A_{w} f\right)_{l\left(V_{0}\right)}^{2}}{a_{j}^{(w)}} .
\end{aligned}
$$

We now consider the following rather restrictive condition.

(A3) $D^{2}=-\gamma D$ for some $\gamma>0$. In other words, all the eigenvalues of $D$ are either 0 or $-\gamma$.

In Example 2.1, (A3) holds with $\gamma=3$.

The following proposition was proved in [1, Theorem 6.1] for the case of the standard Dirichlet form on $S G_{2}$; however, that proof was different from the one presented here. 
Proposition 2.9. Assume the conditions (A1)-(A3). For each $w \in W_{*}$, there exists a set of positive numbers $\left\{b_{j}^{(w)}\right\}_{j \in S_{0}}$ such that

$$
\frac{\nu_{f}\left(K_{w}\right)}{\nu\left(K_{w}\right)}=\sum_{j \in S_{0}} b_{j}^{(w)} \frac{d \nu_{f}}{d \nu}\left(w j^{\infty}\right), \quad f \in \mathcal{H} .
$$

If $\# V_{0} \geq 3$, the $\left\{b_{j}^{(w)}\right\}_{j \in S_{0}}$ are uniquely determined.

Proof. Since ${ }^{t} D D=D^{2}=-\gamma D$,

$$
\begin{aligned}
\frac{\nu_{f}\left(K_{w}\right)}{\nu\left(K_{w}\right)} & =\frac{{ }^{t}\left(A_{w} f\right) D\left(A_{w} f\right)}{\sum_{k=1}^{N}{ }^{t}\left(A_{w} h_{k}\right) D\left(A_{w} h_{k}\right)} \\
& =\frac{-\gamma^{-1} \cdot{ }^{t}\left(D A_{w} f\right)\left(D A_{w} f\right)}{-\gamma^{-1} \sum_{k=1}^{N}{ }^{t}\left(D A_{w} h_{k}\right)\left(D A_{w} h_{k}\right)} \\
& =\frac{\sum_{j \in S_{0}}\left(u_{j}, A_{w} f\right)_{l\left(V_{0}\right)}^{2}}{\sum_{k=1}^{N} \sum_{i \in S_{0}}\left(u_{i}, A_{w} h_{k}\right)_{l\left(V_{0}\right)}^{2}} \\
& =\sum_{j \in S_{0}} \frac{a_{j}^{(w)}}{\sum_{i \in S_{0}} a_{i}^{(w)}} \frac{d \nu_{f}}{d \nu}\left(w j^{\infty}\right) .
\end{aligned}
$$

Therefore, (2.3) holds by letting

$$
b_{j}^{(w)}=\frac{a_{j}^{(w)}}{\sum_{i \in S_{0}} a_{i}^{(w)}} .
$$

To prove the uniqueness of $\left\{b_{j}^{(w)}\right\}_{j \in S_{0}}$, it suffices to prove that

$$
\sum_{j \in S_{0}} \beta_{j}\left(u_{j}, A_{w} f\right)_{l\left(V_{0}\right)}^{2}=0 \quad \text { for all } f \in \mathcal{H}
$$

implies $\beta_{j}=0$ for all $j \in S_{0}$. Let $j$ and $k$ be distinct elements of $S_{0}$. Denote the fixed points of $\psi_{j}$ and $\psi_{k}$ by $p_{j}$ and $p_{k}$, respectively. From Lemma 2.2, there exists an $x \in l\left(V_{0}\right)$ such that $(D x)\left(p_{j}\right)=1,(D x)\left(p_{k}\right)=-1$, and $(D x)(p)=0$ for $p \in V_{0} \backslash\left\{p_{j}, p_{k}\right\}$; in other words, $\left(u_{j}, x\right)_{l\left(V_{0}\right)}=1,\left(u_{k}, x\right)_{l\left(V_{0}\right)}=-1$, and $\left(u_{i}, x\right)_{l\left(V_{0}\right)}=0$ for $i \in S_{0} \backslash\{j, k\}$. From the surjectiveness of $A_{w}$, there exists an $f \in \mathcal{H} \simeq l\left(V_{0}\right)$ such that $A_{w} f=x$. Then, from (2.5), $\beta_{j}+\beta_{k}=0$. This relation implies that $\beta_{j}=0$ for all $j \in S_{0}$ because $\# S_{0}=\# V_{0} \geq 3$.

From (2.4), we have the identity

$$
\sum_{j \in S_{0}} b_{j}^{(w)}=1
$$


The coefficients $\left\{b_{j}^{(w)}\right\}_{j \in S_{0}}$ provide some information on the distribution of the energy measures of harmonic functions. In a typical example, $\left\{b_{j}^{(w)}\right\}_{j \in S_{0}}$ describes the skewness of $\nu$ on the cell $K_{w j}$ relative to $K_{w}$ as follows, which is due to Bell, Ho, and Strichartz [1].

Theorem 2.10 (cf. [1, Theorem 6.3]). We consider the standard Dirichlet form on $S G_{2}$ given in Example 2.1 and write $S=S_{0}=\{1,2,3\}$. As a choice of $\left\{h_{i}\right\}_{i=1}^{N} \subset \mathcal{H}$, let $N=2$ and take a pair $h_{1}, h_{2} \in \mathcal{H}$ so that $\mathcal{E}\left(h_{i}, h_{j}\right)=\delta_{i j} / 4$ for any $i, j \in\{1,2\}$, where $\delta_{i j}$ represents the Kronecker delta. Accordingly, $\nu=\nu_{h_{1}}+\nu_{h_{2}}$. Then, the identity

$$
\frac{1}{5}\left(b_{j}^{(w)}-\frac{1}{3}\right)=\frac{1}{4}\left(\frac{\nu\left(K_{w j}\right)}{\nu\left(K_{w}\right)}-\frac{1}{3}\right)
$$

holds for any $w \in W_{*}$ and $j \in S_{0}$.

It is easy to see that the measure $\nu$ given above is a probability measure on $K$ and is independent of the choice of $h_{1}$ and $h_{2}$. For $S G_{l}$ with $l \geq 3$, such a clear interpretation of $\left\{b_{j}^{(w)}\right\}_{j \in S_{0}}$ as in Theorem 2.10 seems difficult to obtain.

Let $\lambda$ be a Borel probability measure on $\Sigma=S^{\mathbb{N}}$, defined as the infinite product of the uniform probability measure $(\# S)^{-1} \sum_{j \in S} \delta_{j}$ on $S$. For $\omega=\omega_{1} \omega_{2} \cdots \in \Sigma$ and $m \in \mathbb{N}$, $[\omega]_{m}$ denotes $\omega_{1} \omega_{2} \cdots \omega_{m} \in W_{m}$. For $m \in \mathbb{Z}_{+}$, let $\xi_{m}$ denote the image measure of $\lambda$ by the map $\Sigma \ni \omega \mapsto\left\{b_{j}^{\left([\omega]_{m}\right)}\right\}_{j \in S} \in \mathbb{R}^{\# S}$. Bell, Ho, and Strichartz [1] discuss some properties of $\xi_{m}$ for the canonical Dirichlet form on $S G_{2}$ and posed conjectures, which we call Conjecture 2.12 below.

Until the end of this section, we consider the Dirichlet form for two-dimensional standard Sierpinski gasket $S G_{2}=: K$ given in Example 2.1 and take the measure $\nu$ as in Theorem 2.10. We write $S=S_{0}=\{1,2,3\}$ and

$$
\begin{aligned}
& \mathbb{D}:=\left\{\left(b_{1}, b_{2}, b_{3}\right) \in \mathbb{R}^{3} \mid \sum_{j=1}^{3} b_{j}=1 \text { and } \sum_{j=1}^{3}\left(b_{j}-\frac{1}{3}\right)^{2}<\frac{1}{6}\right\} \\
& \left(=\left\{\left(b_{1}, b_{2}, b_{3}\right) \in \mathbb{R}^{3} \mid \sum_{j=1}^{3} b_{j}=1 \text { and } \sum_{j=1}^{3} b_{j}^{2}<\frac{1}{2}\right\}\right) .
\end{aligned}
$$

Theorem 2.11 (cf. [1, Theorem 6.5]). For all $w \in W_{*},\left(b_{1}^{(w)}, b_{2}^{(w)}, b_{3}^{(w)}\right)$ belongs to $\mathbb{D}$; that is,

$$
\sum_{j=1}^{3}\left(b_{j}^{(w)}-\frac{1}{3}\right)^{2}<\frac{1}{6} .
$$

This inequality is sharp. In particular, $\xi_{m}$ concentrates on $\mathbb{D}$ for all $m$.

We note that (2.7) can be rewritten as

$$
\sum_{j=1}^{3}\left(b_{j}^{(w)}\right)^{2}<\frac{1}{2}
$$

because of (2.6). 
Conjecture 2.12 (cf. [1, Conjectures 7.1 and 7.2]). Let $(r, \theta)$ be polar coordinates for the disk $\mathbb{D}$ with center $\boldsymbol{c}=(1 / 3,1 / 3,1 / 3)$. More specifically,

$$
\begin{aligned}
& r(z)=|z-\boldsymbol{c}|_{\mathbb{R}^{3}}, \\
& \theta(z)=\operatorname{Arg}\left(\left(z-\boldsymbol{c}, \boldsymbol{a}_{1}\right)_{\mathbb{R}^{3}}+\sqrt{-1}\left(z-\boldsymbol{c}, \boldsymbol{a}_{2}\right)_{\mathbb{R}^{3}}\right) \in(-\pi, \pi]
\end{aligned}
$$

with $\boldsymbol{a}_{1}=(1 / \sqrt{2},-1 / \sqrt{2}, 0), \boldsymbol{a}_{2}=(-1 / \sqrt{6},-1 / \sqrt{6}, 2 / \sqrt{6})$, where $(\cdot, \cdot)_{\mathbb{R}^{3}}$ and $|\cdot|_{\mathbb{R}^{3}}$ denote the standard inner product and norm on $\mathbb{R}^{3}$, respectively. For $m \in \mathbb{N}$, let $P_{m}$ and $Q_{m}$ denote the image measures of $\xi_{m}$ by the mappings $\theta(\cdot)$ and $r(\cdot)$, respectively. Then:

(1) $P_{m}$ converges weakly to an absolutely continuous measure on $(-\pi, \pi]$ as $m \rightarrow$ $\infty$;

(2) $Q_{m}$ converges weakly to the delta measure at $1 / \sqrt{6}$ as $m \rightarrow \infty$.

Bell, Ho, and Strichartz [1] also conjectured the invariance of the limit of $P_{m}$ under some rational maps, but we skip the details because we do not discuss such kind of property in this paper.

In the next section we prove Theorem 2.11 and confirm Conjecture 2.12(2) in a slightly more general situation.

\section{Main results}

We keep the notation used in the previous section and always assume conditions (A1)-(A3).

Fix $w \in W_{*}$. For $j \in S_{0}$, let $z_{j}={ }^{t} A_{w} u_{j}$. Note that $z_{j} \in \tilde{l}\left(V_{0}\right)$ and $z_{j} \neq 0$ from Lemma 2.7. Also, since $\sum_{j \in S_{0}} u_{j}=0$ from $D \mathbf{1}=0$, we have

$$
\sum_{j \in S_{0}} z_{j}=0
$$

For $x, y \in \tilde{l}\left(V_{0}\right) \subset l\left(V_{0}\right)$, we define

$$
\langle x, y\rangle=\sum_{k=1}^{N}\left(x, h_{k}\right)_{l\left(V_{0}\right)}\left(y, h_{k}\right)_{l\left(V_{0}\right)} \quad \text { and } \quad\|x\|=\langle x, x\rangle^{1 / 2} .
$$

Then, $\langle\cdot, \cdot\rangle$ is an inner product on $\tilde{l}\left(V_{0}\right)$ and the identity $\left\|z_{j}\right\|^{2}=a_{j}^{(w)}$ holds. We remark that there exists a positive definite symmetric operator $H$ on $\tilde{l}\left(V_{0}\right)$ such that $\langle x, y\rangle=(H x, H y)_{l\left(V_{0}\right)}$ for all $x, y \in \tilde{l}\left(V_{0}\right)$.

Fix an arbitrary $k \in S_{0}$ and let $S_{0}^{\prime}=S_{0} \backslash\{k\}$. Then we have the following lemma. 
Lemma 3.1. The following identity holds:

$$
\sum_{j \in S_{0}}\left(b_{j}^{(w)}\right)^{2}=\left(2+\frac{2 \sum_{i, j \in S_{0}^{\prime}, i \neq j}\left\|z_{i}\right\|^{2}\left\|z_{j}\right\|^{2}-\left(\sum_{i, j \in S_{0}^{\prime}, i \neq j}\left\langle z_{i}, z_{j}\right\rangle\right)^{2}}{\sum_{j \in S_{0}}\left\|z_{j}\right\|^{4}}\right)^{-1} .
$$

Proof. From (2.4),

$$
\begin{aligned}
\sum_{j \in S_{0}}\left(b_{j}^{(w)}\right)^{2} & =\frac{\sum_{j \in S_{0}}\left(a_{j}^{(w)}\right)^{2}}{\left(\sum_{j \in S_{0}} a_{j}^{(w)}\right)^{2}}=\frac{\sum_{j \in S_{0}}\left\|z_{j}\right\|^{4}}{\left(\sum_{j \in S_{0}}\left\|z_{j}\right\|^{2}\right)^{2}} \\
& =\left(2+\frac{\left(\sum_{j \in S_{0}}\left\|z_{j}\right\|^{2}\right)^{2}-2 \sum_{j \in S_{0}}\left\|z_{j}\right\|^{4}}{\sum_{j \in S_{0}}\left\|z_{j}\right\|^{4}}\right)^{-1} .
\end{aligned}
$$

Using the identity $z_{k}=-\sum_{j \in S_{0}^{\prime}} z_{j}$ from (3.1), we have

$$
\begin{aligned}
\sum_{j \in S_{0}}\left\|z_{j}\right\|^{4}= & \sum_{j \in S_{0}^{\prime}}\left\|z_{j}\right\|^{4}+\left\|\sum_{j \in S_{0}^{\prime}} z_{j}\right\|^{4} \\
= & \sum_{j \in S_{0}^{\prime}}\left\|z_{j}\right\|^{4}+\left(\sum_{j \in S_{0}^{\prime}}\left\|z_{j}\right\|^{2}+\sum_{i, j \in S_{0}^{\prime}, i \neq j}\left\langle z_{i}, z_{j}\right\rangle\right)^{2} \\
= & 2 \sum_{j \in S_{0}^{\prime}}\left\|z_{j}\right\|^{4}+\sum_{i, j \in S_{0}^{\prime}, i \neq j}\left\|z_{i}\right\|^{2}\left\|z_{j}\right\|^{2}+2 \sum_{j \in S_{0}^{\prime}}\left\|z_{j}\right\|^{2} \sum_{i, j \in S_{0}^{\prime}, i \neq j}\left\langle z_{i}, z_{j}\right\rangle \\
& +\left(\sum_{i, j \in S_{0}^{\prime}, i \neq j}\left\langle z_{i}, z_{j}\right\rangle\right)^{2}
\end{aligned}
$$

and

$$
\begin{aligned}
& \left(\sum_{j \in S_{0}}\left\|z_{j}\right\|^{2}\right)^{2}-\sum_{j \in S_{0}}\left\|z_{j}\right\|^{4} \\
& =\sum_{i, j \in S_{0}, i \neq j}\left\|z_{i}\right\|^{2}\left\|z_{j}\right\|^{2} \\
& =\sum_{i, j \in S_{0}^{\prime}, i \neq j}\left\|z_{i}\right\|^{2}\left\|z_{j}\right\|^{2}+2\left\|\sum_{j \in S_{0}^{\prime}} z_{j}\right\|^{2} \sum_{j \in S_{0}^{\prime}}\left\|z_{j}\right\|^{2} \\
& =\sum_{i, j \in S_{0}^{\prime}, i \neq j}\left\|z_{i}\right\|^{2}\left\|z_{j}\right\|^{2}+2\left(\sum_{j \in S_{0}^{\prime}}\left\|z_{j}\right\|^{2}\right)^{2}+2 \sum_{i, j \in S_{0}^{\prime}, i \neq j}\left\langle z_{i}, z_{j}\right\rangle \sum_{j \in S_{0}^{\prime}}\left\|z_{j}\right\|^{2}
\end{aligned}
$$




$$
=3 \sum_{i, j \in S_{0}^{\prime}, i \neq j}\left\|z_{i}\right\|^{2}\left\|z_{j}\right\|^{2}+2 \sum_{j \in S_{0}^{\prime}}\left\|z_{j}\right\|^{4}+2 \sum_{i, j \in S_{0}^{\prime}, i \neq j}\left\langle z_{i}, z_{j}\right\rangle \sum_{j \in S_{0}^{\prime}}\left\|z_{j}\right\|^{2} .
$$

Then,

$$
\left(\sum_{j \in S_{0}}\left\|z_{j}\right\|^{2}\right)^{2}-2 \sum_{j \in S_{0}}\left\|z_{j}\right\|^{4}=2 \sum_{i, j \in S_{0}^{\prime}, i \neq j}\left\|z_{i}\right\|^{2}\left\|z_{j}\right\|^{2}-\left(\sum_{i, j \in S_{0}^{\prime}, i \neq j}\left\langle z_{i}, z_{j}\right\rangle\right)^{2}
$$

by combining (3.4) and (3.5). This identity and (3.3) imply (3.2).

Lastly we consider the following condition.

(A4) $\# V_{0}=3$.

The following extends Theorem 2.11 ([1, Theorem 6.5]) to more general situations, and the proof is more straightforward.

Theorem 3.2. Under the conditions (A1)-(A4),

$$
\sum_{j \in S_{0}}\left(b_{j}^{(w)}\right)^{2}<\frac{1}{2}
$$

for all $w \in W_{*}$. This inequality is sharp.

Proof. Let $S_{0}^{\prime}=\{1,2\}$. Then, (3.2) can be rewritten as

$$
\sum_{j \in S_{0}}\left(b_{j}^{(w)}\right)^{2}=\left(2+4 \cdot \frac{\left\|z_{1}\right\|^{2}\left\|z_{2}\right\|^{2}-\left\langle z_{1}, z_{2}\right\rangle^{2}}{\sum_{j \in S_{0}}\left\|z_{j}\right\|^{4}}\right)^{-1} .
$$

Moreover, the inequality $\left|\left\langle z_{1}, z_{2}\right\rangle\right| \leq\left\|z_{1}\right\|\left\|z_{2}\right\|$ holds with equality if and only if $z_{1}$ and $z_{2}$ are linearly dependent. Since $u_{1}$ and $u_{2}$ are linearly independent by the property (D2) of $D$, the inequality is strict. Therefore, we obtain (3.6). The sharpness of this inequality is confirmed by Theorem 3.6 below, so we omit the proof here.

Remark 3.3. As can be seen from the proof above, it seems difficult to obtain a good estimate of $\sum_{j \in S_{0}}\left(b_{j}^{(w)}\right)^{2}$ if $\# V_{0}>3$. Indeed, if $\# V_{0}=4$ and $S_{0}=\{1,2,3,4\}$, Eq. (3.2) is rewritten as

$$
\sum_{j \in S_{0}}\left(b_{j}^{(w)}\right)^{2}=\left(2+4 \cdot \frac{I}{\sum_{j \in S_{0}}\left\|z_{j}\right\|^{4}}\right)^{-1}
$$

with

$$
\begin{aligned}
I= & \sum_{(i, j) \in\{(1,2),(2,3),(3,1)\}}\left(\left\|z_{i}\right\|^{2}\left\|z_{j}\right\|^{2}-\left\langle z_{i}, z_{j}\right\rangle^{2}\right) \\
& -2 \sum_{(i, j, k) \in\{(1,2,3),(2,3,1),(3,1,2)\}}\left\langle z_{i}, z_{j}\right\rangle\left\langle z_{j}, z_{k}\right\rangle .
\end{aligned}
$$

We may need other functionals to specify the range of $\left\{b_{j}^{(w)}\right\}_{j \in S_{0}}$ in such a case. 
Let $\tilde{D}$ denote the restriction of $D$ as a negative definite symmetric operator on $\tilde{l}\left(V_{0}\right)$. For $w \in W_{*}$, let $\tilde{A}_{w}$ denote the restriction of $P A_{w}\left(=P A_{w} P\right)$ as a linear operator on $\tilde{l}\left(V_{0}\right)$.

For the statement of the main theorem, we recall the concept of strong irreducibility of random matrices.

Definition 3.4. A set $\mathcal{T}$ of invertible linear operators on $\tilde{l}\left(V_{0}\right)$ is called strongly irreducible if there does not exist a finite family $L_{1}, \ldots, L_{k}$ of proper linear subspaces of $\tilde{l}\left(V_{0}\right)$ such that $M\left(L_{1} \cup \cdots \cup L_{k}\right)=L_{1} \cup \cdots \cup L_{k}$ for all $M \in \mathcal{T}$.

Example 3.5. We again consider a canonical harmonic structure on the twodimensional level $l$ Sierpinski gasket in Example 2.1. Further, we assume (A2). Then we can prove that $\left\{\tilde{A}_{i}\right\}_{i \in S}$ is strongly irreducible. Indeed, from (A2) and the fact that the sequence $\left\{\left(\left|\operatorname{det} \tilde{A}_{j}\right|^{-1 / 2} \tilde{A}_{j}\right)^{n}\right\}_{n=1}^{\infty}$ is unbounded for $j \in S_{0}$, as shown in the proof of Theorem 3.6 below, it suffices to prove the following claim by [3, Part A, Chapter II, Proposition 4.3]:

For every $x \in \tilde{l}\left(V_{0}\right) \backslash\{0\}$, the set $\left\{\tilde{A}_{i}^{n} x \mid i \in S, n \in \mathbb{Z}_{+}\right\}$has three elements $y_{1}, y_{2}, y_{3}$ such that $y_{j}$ and $y_{k}$ are pairwise linearly independent for $j \neq k$.

Let $S_{0}=\{1,2,3\}$. From the symmetry of the harmonic structure and Lemma 2.4(1), $\tilde{A}_{1}$ has two different eigenvalues and the eigenvectors of $\tilde{A}_{1}$ (up to multiplicative constants) are $z_{1}:={ }^{t}(2,-1,-1)$ and $z_{2}:={ }^{t}(0,1,-1)$. The set of eigenvectors of $\tilde{A}_{1}^{2}$ is the same as that of $\tilde{A}_{1}$. The same claims hold for $\tilde{A}_{2}$ with $z_{1}$ and $z_{2}$ replaced by ${ }^{t}(-1,2,-1)$ and ${ }^{t}(1,0,-1)$, respectively. Now, let $x \in \tilde{l}\left(V_{0}\right) \backslash\{0\}$. If $x$ and $z_{i}$ are linearly dependent for $i=1$ or 2 , any two of $\left\{x, A_{2} x, A_{2}^{2} x\right\}$ are linearly independent. Otherwise, any two of $\left\{x, A_{1} x, A_{1}^{2} x\right\}$ are linearly independent. Therefore, (3.8) follows.

We now resume our discussion of the general situation. The following is the main theorem of this paper.

Theorem 3.6. Assume the conditions (A1)-(A4). Let $\kappa$ be a Borel measure on $\Sigma=S^{\mathbb{N}}$. We further suppose either of the following cases.

(I) $\kappa$ is an infinite product of a probability measure on $S$ with full support, and $\left\{\tilde{A}_{i}\right\}_{i \in S}$ is strongly irreducible.

(II) The image measure of $\kappa$ by $\pi: \Sigma \rightarrow K$ is absolutely continuous with respect to $\nu$.

Then,

$$
\lim _{n \rightarrow \infty} \sum_{j \in S_{0}}\left(b_{j}^{\left([\omega]_{n}\right)}\right)^{2}=\frac{1}{2} \quad \text { for } \kappa \text {-a.e. } \omega .
$$

In particular, the image measure of $\kappa$ by the map $\Sigma \ni \omega \mapsto\left\{b_{j}^{\left([\omega]_{n}\right)}\right\}_{j \in S_{0}} \in$ $l\left(S_{0}\right) \cong l\left(V_{0}\right)$ converges weakly as $n \rightarrow \infty$ to a measure that concentrates on the set $\left\{x \in l\left(V_{0}\right) \mid \sum_{p \in V_{0}} x(p)=1\right.$ and $\left.|x|_{l\left(V_{0}\right)}^{2}=1 / 2\right\}$. 
The result for Case (I) gives an affirmative answer to Conjecture 2.12(2). We remark that the strong irreducibility of $\left\{\tilde{A}_{i}\right\}_{i \in S}$ is not necessary in Case (II).

Proof of Theorem 3.6. First, we note that $\tilde{l}\left(V_{0}\right)$ is two-dimensional because of (A4). Using the same notation as in Theorem 3.2, with $w=[\omega]_{n}$ for $\omega \in \Sigma$ and $n \in \mathbb{N}$,

$$
\begin{aligned}
\left\|z_{1}\right\|^{2}\left\|z_{2}\right\|^{2}-\left\langle z_{1}, z_{2}\right\rangle^{2} & =\left|H z_{1}\right|_{l\left(V_{0}\right)}^{2}\left|H z_{2}\right|_{l\left(V_{0}\right)}^{2}-\left(H z_{1}, H z_{2}\right)_{l\left(V_{0}\right)}^{2} \\
& =\left|H z_{1} \wedge H z_{2}\right|^{2} \bigwedge^{2} \tilde{l}\left(V_{0}\right) \\
& =(\operatorname{det} H)^{2}\left(\operatorname{det} \tilde{A}_{[\omega]_{n}}\right)^{2}\left|u_{1} \wedge u_{2}\right|_{\wedge^{2} \tilde{l}\left(V_{0}\right)}^{2} .
\end{aligned}
$$

Moreover, since $u_{1}$ and $u_{2}$ are linearly independent, the map $B \mapsto\left(\left\|B u_{1}\right\|^{4}+\right.$ $\left.\left\|B u_{2}\right\|^{4}\right)^{1 / 4}$ provides a norm on the space $\mathcal{L}\left(\tilde{l}\left(V_{0}\right)\right)$ of all linear operators on $\tilde{l}\left(V_{0}\right)$. Therefore,

$$
\sum_{j \in S_{0}}\left\|z_{j}\right\|^{4} \geq\left\|\tilde{A}_{[\omega]_{n}} u_{1}\right\|^{4}+\left\|\tilde{A}_{[\omega]_{n}} u_{2}\right\|^{4} \geq c\left\|\tilde{A}_{[\omega]_{n}}\right\|_{\mathrm{op}}^{4}
$$

where $\|\cdot\|_{\text {op }}$ represents the operator norm on $\mathcal{L}\left(\tilde{l}\left(V_{0}\right)\right)$ and $c$ is a positive constant independent of $\omega$ and $n$. Then

$$
0<\frac{\left\|z_{1}\right\|^{2}\left\|z_{2}\right\|^{2}-\left\langle z_{1}, z_{2}\right\rangle^{2}}{\sum_{j \in S_{0}}\left\|z_{j}\right\|^{4}} \leq \frac{(\operatorname{det} H)^{2}\left(\operatorname{det} \tilde{A}_{[\omega]_{n}}\right)^{2}\left|u_{1} \wedge u_{2}\right|_{\Lambda^{2}}^{2} \tilde{l}\left(V_{0}\right)}{c\left\|\tilde{A}_{[\omega]_{n}}\right\|_{\text {op }}^{4}} .
$$

By virtue of (3.7), Eq. (3.9) follows if we can prove that

$$
\lim _{n \rightarrow \infty} \frac{\left(\operatorname{det} \tilde{A}_{[\omega]_{n}}\right)^{2}}{\left\|\tilde{A}_{[\omega]_{n}}\right\|_{\mathrm{op}}^{4}}=0 \quad \text { for } \kappa \text {-a.e. } \omega \text {. }
$$

Suppose Case (I) and fix $j \in S_{0}$. Since $\tilde{A}_{j}$ is invertible from (A2) and has two eigenvalues with different moduli from Lemma 2.4(1), the sequence $\left\{\left(\left|\operatorname{det} \tilde{A}_{j}\right|^{-1 / 2} \tilde{A}_{j}\right)^{n}\right\}_{n=1}^{\infty}$ is unbounded. Together with strong irreducibility of $\left\{A_{i}\right\}_{i \in S}$, Furstenberg's theorem (cf. [3, Part A, Chapter II, Theorems 4.1 and 3.6]) implies that for two elements $x_{1}, x_{2} \in \tilde{l}\left(V_{0}\right)$ which are linearly independent,

$$
\lim _{n \rightarrow \infty} \delta\left(\tilde{A}_{[\omega]_{n}} x_{1}, \tilde{A}_{[\omega]_{n}} x_{2}\right)=0 \text { for } \kappa \text {-a.e. } \omega \text {. }
$$

Here, $\delta(\cdot, \cdot) \in[0,1]$ denotes the angular distance, that is,

$$
\delta\left(y_{1}, y_{2}\right)=\sqrt{1-\left(\frac{y_{1}}{\left|y_{1}\right|_{l\left(V_{0}\right)}}, \frac{y_{2}}{\left|y_{2}\right| l\left(V_{0}\right)}\right)_{l\left(V_{0}\right)}^{2}} \text { for } y_{1}, y_{2} \in \tilde{l}\left(V_{0}\right) \backslash\{0\} .
$$

Let $x_{1}, x_{2}$ be an orthonormal basis of the inner product space $\left(\tilde{l}\left(V_{0}\right),(\cdot, \cdot)_{l\left(V_{0}\right)}\right)$. Then

$$
\delta\left(\tilde{A}_{[\omega]_{n}} x_{1}, \tilde{A}_{[\omega]_{n}} x_{2}\right)=\frac{\left|\operatorname{det} \tilde{A}_{[\omega]_{n}}\right|}{\left|\tilde{A}_{[\omega]_{n}} x_{1}\right|_{l\left(V_{0}\right)}\left|\tilde{A}_{[\omega]_{n}} x_{2}\right|_{l\left(V_{0}\right)}} \geq \frac{\left|\operatorname{det} \tilde{A}_{[\omega]_{n}}\right|}{\left\|\tilde{A}_{[\omega]_{n}}\right\|_{\mathrm{op}}^{2}}
$$


Thus we obtain (3.10).

Next, suppose Case (II). Again let $x_{1}, x_{2}$ be an orthonormal basis of $\tilde{l}\left(V_{0}\right)$. Define $\hat{h}_{i}=\iota\left(x_{i}\right)$ for $i=1,2$ and $\hat{\nu}=\nu_{\hat{h}_{1}}+\nu_{\hat{h}_{2}}$. Since the linear span of $\hat{h}_{1}$, $\hat{h}_{2}$, and constant functions is $\mathcal{H}, \nu$ and $\hat{\nu}$ are mutually absolutely continuous from Lemma 2.6. Therefore, we may assume that the image measure of $\kappa$ by the mapping $\pi$ coincides with $\hat{\nu}$ for proving $(3.10){ }^{\dagger}$ In our situation, the index ([9, Definition 2.9]) of the Dirichlet form under consideration is 1 (see [8, Proposition 3.4] or [10, Theorem 4.10] for the proof; see also [14]). In particular, we have

$$
\operatorname{rank}\left(\frac{d \nu_{\hat{h}_{i}, \hat{h}_{j}}}{d \hat{\nu}}\right)_{i, j=1}^{2} \leq 1 \quad \hat{\nu} \text {-a.e. } \ddagger
$$

On the other hand, for $i, j \in\{1,2\}$,

$$
\frac{d \nu_{\hat{h}_{i}, \hat{h}_{j}}}{d \hat{\nu}}(\pi(\omega))=\lim _{n \rightarrow \infty} \frac{\nu_{\hat{h}_{i}, \hat{h}_{j}}\left(K_{[\omega]_{n}}\right)}{\hat{\nu}\left(K_{[\omega]_{n}}\right)}=\lim _{n \rightarrow \infty} \frac{-{ }^{t} x_{i}{ }^{t} \tilde{A}_{[\omega]_{n}} \tilde{D} \tilde{A}_{[\omega]_{n}} x_{j}}{\operatorname{tr}\left(-{ }^{t} \tilde{A}_{[\omega]_{n}} \tilde{D}_{[\omega]_{n}}\right)} \text { for } \kappa \text {-a.e. } \omega
$$

where the first equality follows from the martingale convergence theorem and the second one is due to Lemma 2.3. Then, for $\kappa$-a.e. $\omega$,

$$
\begin{aligned}
0 & =\lim _{n \rightarrow \infty} \operatorname{det}\left(\frac{-{ }^{t} \tilde{A}_{[\omega]_{n}} \tilde{D} \tilde{A}_{[\omega]_{n}}}{\operatorname{tr}\left(-{ }^{t} \tilde{A}_{[\omega]_{n}} \tilde{D} \tilde{A}_{[\omega]_{n}}\right)}\right) \\
& =\lim _{n \rightarrow \infty} \frac{\left(\operatorname{det} \tilde{A}_{[\omega]_{n}}\right)^{2} \operatorname{det}(-\tilde{D})}{\left\|\sqrt{-\tilde{D}} \tilde{A}_{[\omega]_{n}}\right\|_{\mathrm{HS}}^{4}} \\
& \geq \varlimsup_{n \rightarrow \infty} \frac{\left(\operatorname{det} \tilde{A}_{[\omega]_{n}}\right)^{2} \operatorname{det}(-\tilde{D})}{c^{\prime}\left\|\tilde{A}_{[\omega]_{n}}\right\|_{\mathrm{op}}^{4}}
\end{aligned}
$$

where $\|\cdot\|_{\text {HS }}$ denotes the Hilbert-Schmidt norm on $\mathcal{L}\left(\tilde{l}\left(V_{0}\right)\right)$ and $c^{\prime}$ is a positive constant depending only on $D$. Thus, (3.10) holds.

The last claim of the theorem follows from the general fact that almost sure convergence implies convergence in law.

\section{Concluding remarks}

We give some comments as concluding remarks.

\footnotetext{
†Since $\hat{\nu}\left(V_{*}\right)=0$, such a $\kappa$ is uniquely identified. More specifically, for $m \in \mathbb{N}$ and $A \subset W_{m}$, $\kappa\left(\left\{\omega \in \Sigma \mid[\omega]_{m} \in A\right\}\right)$ is given by $\sum_{k=1}^{2} \sum_{w \in A} 2 r_{w}^{-1} \mathcal{E}\left(\hat{h}_{k} \circ \psi_{w}, \hat{h}_{k} \circ \psi_{w}\right)$ from Lemma 2.3.

${ }^{\ddagger}$ In fact, the equality holds from [9, Proposition 2.11].
} 

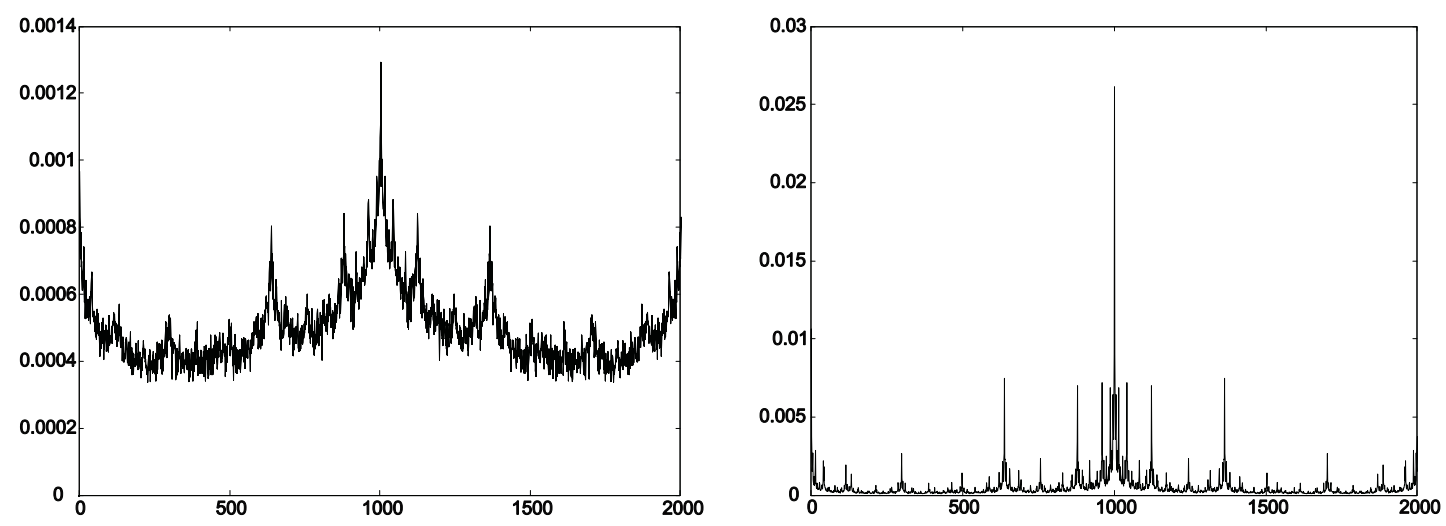

Figure 2. Angular distributions

(1) As can be seen from the proof, Condition (A4) is crucial for Theorem 3.2 and thus for Theorem 3.6. It may be an interesting problem to provide an appropriate formulation when $\# V_{0}>3$.

(2) In both cases (I) and (II) in Theorem 3.6, $\kappa$ has no mass on $\pi^{-1}\left(V_{*}\right)$. Therefore, the statements of Theorem 3.6 and Conjecture 2.12 can be rephrased in terms of a measure on $K$ instead of the measure $\kappa$ on $\Sigma$ : that is, self-similar measures on $K$ in Case (I) and $\nu$ in Case (II), respectively.

(3) In Theorem 3.6, the measure $\kappa$ of Case (I) and that of Case (II) are mutually singular in many cases (cf. [12, Theorem 2]). Case (II) looks like a more natural formulation in the sense that there is no need for the extra assumption of the strong irreducibility of $\left\{A_{j}\right\}_{j \in S}$ and because the concept of the index of Dirichlet forms, which also has probabilistic interpretations [9, 11], appears naturally in the proof.

(4) At the moment, there are no clues concerning Conjecture 2.12(1). The distribution of $P_{m}$ with $m=13$ is given in the left-hand graph of Figure 2. This figure shows the normalized histogram (2000 slices) of $P_{13}$ on $[-\pi / 3, \pi / 3]$ for the Dirichlet form on $S G_{2}$ assumed in Conjecture 2.12; because of the

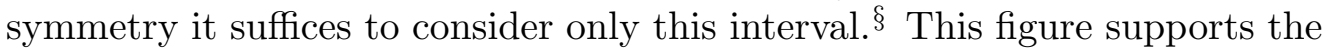
validity of Conjecture 2.12(1). On the other hand, the right-hand graph of Figure 2 shows the distribution on $[-\pi / 3, \pi / 3]$ of the image measure of $\kappa$ by the map $\Sigma \ni \omega \mapsto \theta\left(\left\{b_{j}^{\left([\omega]_{m}\right)}\right\}_{j=1}^{3}\right) \in(-\pi, \pi]$ with $m=13$, where $\kappa$ is taken so that its image measure by the map $\pi: \Sigma \rightarrow K$ is equal to the measure $\nu$ given in Theorem 2.10. Here, the interval $[-\pi / 3, \pi / 3]$ is again divided into 2000 slices. The distribution looks very different and the possible limit measure as $m \rightarrow \infty$ might be singular with respect to the Lebesgue measure.

\footnotetext{
$\S$ The histogram of $P_{13}$ with 100 slices is also provided in [1, Figure 7.4]; the figure there corresponds to the right half of ours. However, with only 100 slices the graph does not seem to reflect the irregular behavior of the distribution of $P_{m}$ very precisely.
} 
M. Hino

\section{References}

[1] R. Bell, C.-W. Ho, and R. S. Strichartz, Energy measures of harmonic functions on the Sierpiński gasket, Indiana Univ. Math. J. 63 (2014), 831-868.

[2] O. Ben-Bassat, R. S. Strichartz and A. Teplyaev, What is not in the domain of the Laplacian on Sierpinski gasket type fractals, J. Funct. Anal. 166 (1999) 197-217.

[3] P. Bougerol and J. Lacroix, Products of random matrices with applications to Schrödinger operators, Birkhäuser, Basel, 1985.

[4] N. Bouleau and F. Hirsch, Dirichlet forms and analysis on Wiener space, de Gruyter Studies in Mathematics 14, Walter de Gruyter, Berlin, 1991.

[5] Z.-Q. Chen and M. Fukushima, Symmetric Markov processes, time change, and boundary theory, London Mathematical Society Monographs Series 35, Princeton University Press, Princeton, NJ, 2012.

[6] M. Fukushima, Y. Oshima and M. Takeda, Dirichlet forms and symmetric Markov processes, Second revised and extended edition, de Gruyter Studies in Mathematics 19, Walter de Gruyter, Berlin, 2011.

[7] M. Hino, On singularity of energy measures on self-similar sets, Probab. Theory Related Fields 132 (2005), 265-290.

[8] M. Hino, Martingale dimensions for fractals, Ann. Probab. 36 (2008), 971-991.

[9] M. Hino, Energy measures and indices of Dirichlet forms, with applications to derivatives on some fractals, Proc. Lond. Math. Soc. (3) 100 (2010), 269-302.

[10] M. Hino, Upper estimate of martingale dimension for self-similar fractals, Probab. Theory Related Fields 156 (2013), 739-793.

[11] M. Hino, Measurable Riemannian structures associated with strong local Dirichlet forms, Math. Nachr. 286 (2013), 1466-1478.

[12] M. Hino and K. Nakahara, On singularity of energy measures on self-similar sets II, Bull. Lond. Math. Soc. 38 (2006), 1019-1032.

[13] J. Kigami, Analysis on fractals, Cambridge Tracts in Mathematics 143, Cambridge University Press, Cambridge, 2001.

[14] S. Kusuoka, Dirichlet forms on fractals and products of random matrices, Publ. Res. Inst. Math. Sci. 25 (1989), 659-680.

Masanori Hino, Graduate School of Engineering Science, Osaka University, Osaka 560-8531, Japan

E-mail: hino@sigmath.es.osaka-u.ac.jp 